Portland State University

PDXScholar

$7-2008$

\title{
Modeling Broadband Ocean Acoustic Transmissions with Time-Varying Sea Surfaces
}

Martin Siderius

Portland State University, siderius@pdx.edu

Michael B. Porter

Follow this and additional works at: https://pdxscholar.library.pdx.edu/ece_fac

Part of the Electrical and Computer Engineering Commons

Let us know how access to this document benefits you.

\section{Citation Details}

Siderius, M., \& Porter, M. B. (2008). Modeling broadband ocean acoustic transmissions with time-varying sea surfaces. Journal of The Acoustical Society of America, 124(1), 137-150.

This Article is brought to you for free and open access. It has been accepted for inclusion in Electrical and Computer Engineering Faculty Publications and Presentations by an authorized administrator of PDXScholar. Please contact us if we can make this document more accessible: pdxscholar@pdx.edu. 


\title{
Modeling broadband ocean acoustic transmissions with time-varying sea surfaces
}

\author{
Martin Siderius and Michael B. Porter \\ HLS Research Inc., 3366 N. Torrey Pines Ct., Suite 310, La Jolla, California 92037
}

(Received 2 October 2007; revised 11 April 2008; accepted 15 April 2008)

\begin{abstract}
Solutions to ocean acoustic scattering problems are often formulated in the frequency domain, which implies that the surface is "frozen" in time. This may be reasonable for short duration signals but breaks down if the surface changes appreciably over the transmission time. Frequency domain solutions are also impractical for source-receiver ranges and frequency bands typical for applications such as acoustic communications (e.g. hundreds to thousands of meters, $1-50 \mathrm{kHz}$ band). In addition, a driving factor in the performance of certain acoustic systems is the Doppler spread, which is often introduced from sea-surface movement. The time-varying nature of the sea surface adds complexity and often leads to a statistical description for the variations in received signals. A purely statistical description likely limits the insight that modeling generally provides. In this paper, time-domain modeling approaches to the sea-surface scattering problem are described. As a benchmark for comparison, the Helmholtz integral equation is used for solutions to static, time-harmonic rough surface problems. The integral equation approach is not practical for time-evolving rough surfaces and two alternatives are formulated. The first approach is relatively simple using ray theory. This is followed with a ray-based formulation of the Helmholtz integral equation with a time-domain Kirchhoff approximation.
\end{abstract}

(C) 2008 Acoustical Society of America. [DOI: 10.1121/1.2920959]

PACS number(s): 43.30.Zk, 43.30.Cq, 43.30.Hw, 43.30.Re [SLB] Pages: 137-150

\section{INTRODUCTION}

For many sonar applications, scattering is treated as an effective loss mechanism and Doppler shifts are often ignored. This may be reasonable for certain types of sonar systems, particularly the low frequency ones. However, new underwater acoustic systems, including those for underwater acoustic communications, are sensitive to both scattering losses and Doppler. In particular, channel equalizers used with bandwidth-efficient, phase-coherent communications methods can be extremely sensitive to Doppler spread. Designing these equalizers to compensate for Doppler often presents a substantial challenge. Significant Doppler spread can be introduced simply from the sound interacting with the moving sea surface; however, the effects are much greater when the source and receiver are also in motion. Simulating signals using a physics-based model can greatly aid in the development of new algorithms and provide valuable performance predictions. Two simulation methods for signals that interact with a time-varying, rough sea surface will be described in this paper. First, a simple technique is proposed that includes Doppler effects due to source/receiver and seasurface motion. This is an extension of an earlier work that was developed to simulate active sonar receptions on moving marine mammals. ${ }^{1}$ The need for comparisons as well as determining the limitations of this approach led to the second technique, which uses an implementation of the time-domain Kirchhoff approximation.

This paper is organized as follows. In Sec. II, the Makai experiment is described, which is useful in motivating the problem by presenting measurements of Doppler-sensitive signals that have interacted with the moving sea surface. In
Secs. III and IV, a ray-based approach is used to model moving sources/receivers as well as a slowly varying sea surface. To model interactions from finer scale sea-surface roughness, Sec. V describes the time-harmonic approach using the Helmholtz-Kirchhoff integral equation. This approach is exact for two dimensional problems with a line source. This section also provides an implementation of the Kirchhoff approximation, which is numerically much less demanding than the integral equation. The described methods are developed for line sources but the applications of interest are better modeled with point sources. Therefore, in Sec. VI, the conversion of line source solutions to point source solutions is described. Finally, in Sec. VII, the time-domain solution is developed for rough surfaces that move in time by using the time-domain Kirchhoff approximation.

\section{THE MAKAI EXPERIMENT}

The motivation for the modeling techniques developed here can be illustrated with data collected during the Makai experiment which took place from September 15 to October 1, 2005 near the coast of Kauai, HI. ${ }^{2}$ The site has a coral sand bottom with a fairly flat bathymetry that was nominally $100 \mathrm{~m}$. The water column was variable but typically had a mixed layer depth of 40-60 m and was downward refracting below. The data were measured on September 24th using both stationary and towed sources (from R/V Kilo Moana). The sources were programmable, underwater acoustic modems developed at SPAWAR Systems Center (referred to as the Telesonar Testbeds, T1 and T2). ${ }^{3}$ Signals were received on the AOB array, which is an autonomous system developed at the University of Algarve, Portugal. The AOB is a drifting 


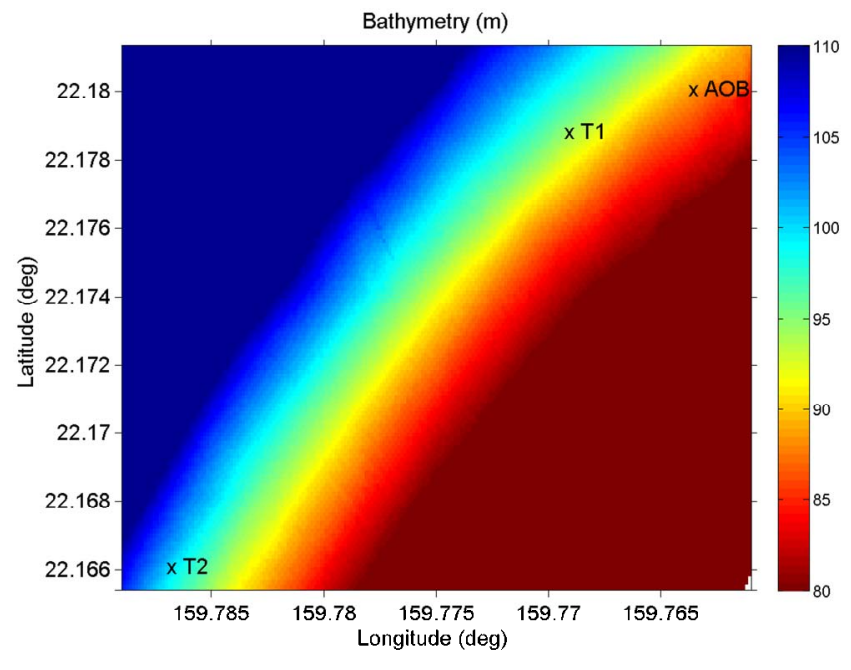

FIG. 1. (Color online) Bathymetry near Kauai with the positions of the AOB vertical array and the Telesonar Testbeds T1 and T2 at 01:00 on JD 268. T1 was about $600 \mathrm{~m}$ away from $\mathrm{AOB}$ and being towed while T2 is about $2.8 \mathrm{~km}$ away and is stationary.

eight-element self-recording array that resembles the size and weight of a standard sonobuoy. ${ }^{4}$ The experiment geometry and bathymetry are shown in Fig. 1. Figure 2 shows a ray trace of the T1-AOB acoustic paths. The paths are numbered on the figure and correspond to (1) direct bounce, (2) surface bounce, (3) bottom bounce, (4) surface-bottom bounce, and (5) bottom-surface bounce. The different path directions have sensitivity to different velocity components. The higher numbered paths are more Doppler sensitive to the vertical velocity components (e.g., from the moving sea surface) and the lower numbered paths (e.g., direct path) are more sensitive to the horizontal velocity components (e.g., from the source or receiver motion).

A binary-phase-shift-keying (BPSK) transmission was used to analyze the channel. ${ }^{5}$ This waveform is commonly used for communication transmissions but for this analysis, it is simply a highly Doppler-sensitive signal that can separate the multipath in time and Doppler spaces. The transmission used cycles of a $9.5 \mathrm{kHz}$ sinusoid with phase shifts introduced to represent a string of 1's and 0's defined by an $m$ sequence. ${ }^{5}$ This signal uses an $m$ sequence (size 1024) with $1500 \mathrm{chips} / \mathrm{s}$. The total length of the sequence was $0.682 \mathrm{~s}$. In a static situation, using a matched filter on this waveform produces an estimate of the channel impulse response. However, in situations with source/receiver and/or sea-surface motion, each path can have a different Doppler shift (due to the angle-dependent propagation paths). A single Doppler

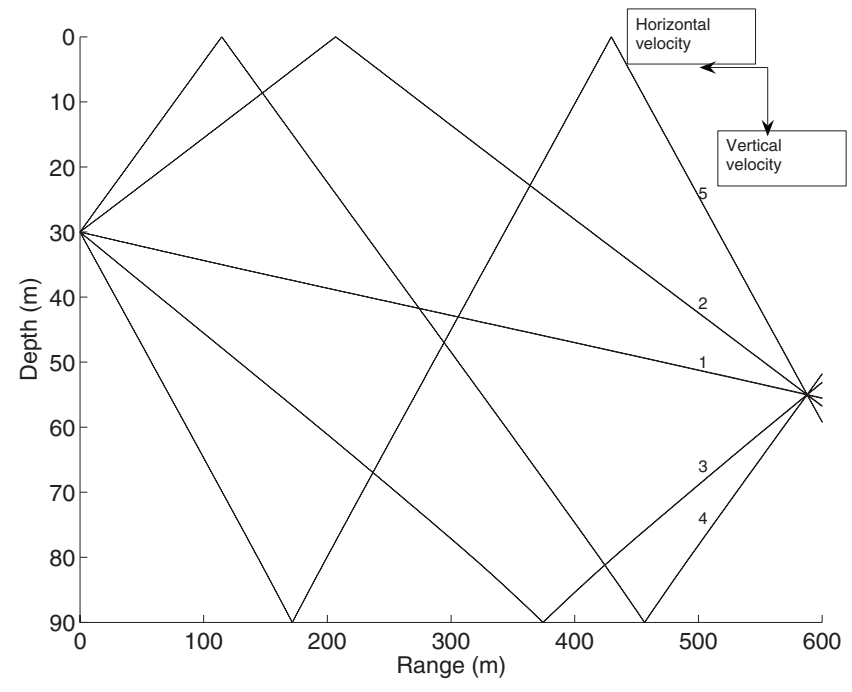

FIG. 2. Ray trace between testbed $\mathrm{T} 1$ and the AOB array. The various paths are labeled (1) direct, (2) surface bounce, (3) bottom bounce, (4) surfacebottom bounce, and (5) bottom-surface bounce.

shift can be applied to the BPSK signal before the matchedfilter process. By sweeping over a variety of shifts, the Doppler for each received arrival can be estimated. The resulting picture provides an estimate of the so-called channel scattering function. ${ }^{5}$ This is referred to as an estimate since a true scattering function requires knowing the continuous time evolution of the impulse response. This is a difficult measurement to make since, in practice, the estimate of the impulse response requires time. The described method to estimate the scattering function does, however, provide the essential information about the relative arrival times of the multipath and how each is Doppler shifted.

Examples of the processed scattering functions at two ranges from the Makai experiment are shown in Fig. 3. Each horizontal trace in the figure results from a matched-filter process using different Doppler-shifted replicas denoted $\tilde{s}_{j}(t)$. The index $j$ corresponds to applied Doppler shifts according to the shift factor $1-v_{j} / c$, where $v_{j}$ is the assumed speed and $c$ is the reference sound speed. The Doppler replicas, $\widetilde{s}_{j}(t)$, are matched filtered against the received time series. ${ }^{6}$ That is,

$$
r_{j}(t)=\int_{+\infty}^{\infty} p(\tau) \widetilde{s}_{j}(\tau-t) d \tau,
$$

where $p(\tau)$ is the received time series and $r_{j}(\tau)$ is the matched-filter output. In this way, $r_{j}(t)$ is indexed over time and Doppler and each multipath arrival produces a peak
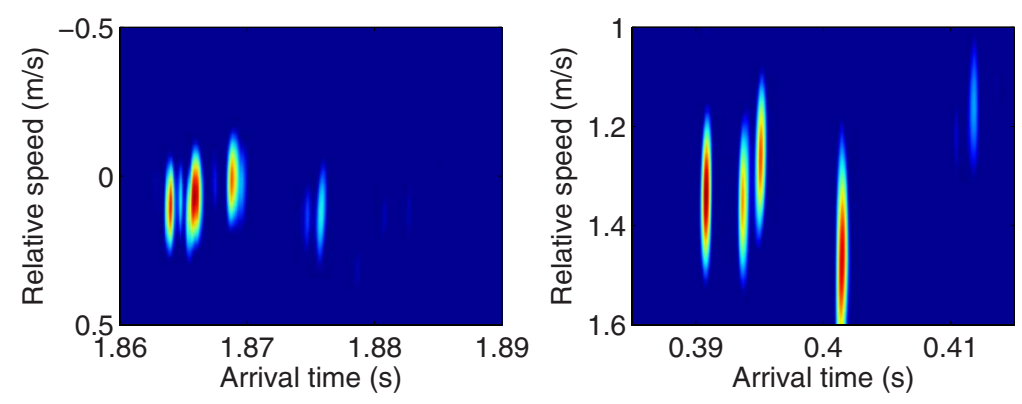

FIG. 3. (Color online) Left panel shows the measured impulse response (or scattering function) for various Doppler shifts indicated on the $y$ axis (as relative speed in $\mathrm{m} / \mathrm{s}$ ) between the drifting AOB and the stationary $\mathrm{T} 2$ at 01:04 on JD 268. Each bright spot corresponds to an arrival with delay time shown along the $x$ axis. Right panel is for a reception from the towed source T1 at 01:02 on JD 268. 
when the Doppler shift of the replica is matched with that arrival.

In the left panel of Fig. 3, the measured scattering function is shown for a $2.8 \mathrm{~km}$ range separation between the fixed Testbed T2 and the drifting AOB. The second measured scattering function is shown in the right panel and is from the towed Testbed T1 and received on the AOB about $600 \mathrm{~m}$ away. The bright spots indicate an arrival in time (i.e., delay time) along the $x$ axis. Note that only the relative time is known so the time series are aligned based on the first arrival and the known distance between the source and receiver. The $y$ axis shows the relative speed (i.e., Doppler) that corresponds to the peaks. The left panel had the stationary source and the Doppler indicates that the AOB was drifting at about $0.1-0.2 \mathrm{~m} / \mathrm{s}$. The estimate from GPS positions indicated about $0.12 \mathrm{~m} / \mathrm{s}$. The first arrivals show decreasing Doppler followed by the last visible arrival having an increased Doppler shift. For horizontal velocity one expects the later arrivals to have decreasing Doppler shifts due to higher propagation angles relative to the direction of motion. The high Doppler on the last arrival implies a component in the vertical which would introduce larger shifts for late arrivals. The right panel of Fig. 3 shows the reception from T1 which was being towed with relative speed between $\mathrm{T} 1$ and the AOB of about $1.2-1.4 \mathrm{~m} / \mathrm{s}$. The estimate from GPS positions is $1.24 \mathrm{~m} / \mathrm{s}$. Like the stationary case, Doppler shifts do not decrease monotonically on the steeper paths but, in some cases, increase. The paths can be identified using the ray trace in Fig. 1. The second and fourth arrivals both have increased Doppler shifts relative to the direct path while the third arrival has a slightly decreased Doppler. Paths 2 and 4 correspond to the surface and surface-bottom bounce paths while the third arrival is the bottom bounce. Note that in both figures, only the first few paths are visible; the later arrivals are more attenuated due to surface/bottom interactions.

In the context of acoustic communications, the spread factor is often used to determine the type of channel and therefore the signaling waveforms. The spread factor is defined as $T_{m} B_{d}$ where $T_{m}$ is the multipath time duration (s) and $B_{d}$ is the Doppler spread $(\mathrm{Hz})$. If the spread factor is less than 1 , the channel is said to be underspread, and if it is greater than 1 it is overspread. It can be highly useful to model the effects that influence the channel spread as this can lead to acoustic communication improvements as well as performance prediction. While the spread factor is a simple metric, it does not give a complete description. For example, does the multipath consist of many arrivals or just a few? The information given in Fig. 3 show not only the multipath delay and Doppler spread but also show the total number and strength of the arrivals. In this example, the spread factor is relatively large but the total number of arrivals is small (i.e., sparse channel). All of these factors are important for communications and correct modeling of these is the primary goal of this work. Modeling techniques that can be applied in estimating the multipath delay and Doppler spread are the topic of the next sections.

\section{MODELING SOURCE AND RECEIVER MOTION WITH RAYS}

In this section, a relatively simple implementation of two dimensional ray methods is extended to treat moving receivers and a moving sea surface. The goals are somewhat similar to the work of Keiffer et $a l^{7}$ but the approach is different, and the emphasis is on broadband signals for application to underwater communications, for example. In a ray formulation, the complex pressure field, $P(\omega)$, can be represented as a sum of $N$ arrival amplitudes $A_{n}(\omega)$ and delays $\tau_{n}(\omega)$ according to

$$
P(\omega)=S(\omega) \sum_{n=1}^{N} A_{n} e^{i \omega \tau_{n}},
$$

where $S(\omega)$ is the spectrum of the source. The specifics of the ray trace algorithm are not critical and there are a number of ray trace models that could have been used here. A summary of most of these type of models can be found from Etter $^{8}$ and Jensen et al. $^{9}$ In the cases considered here, an azimuthally symmetric geometry is assumed and the arrival amplitudes and delays are computed using the two dimensional Gaussian beam implementation in the Bellhop package. $^{10,11}$

According to the convolution theorem, a product of two spectra is a convolution in the time domain. This leads to the corresponding time-domain representation for the received waveform, $p(t)$, which is often written as

$$
p(t)=\sum_{n=1}^{N} A_{n}(t) s\left[t-\tau_{n}(t)\right]
$$

where $s(t)$ is the source waveform. This expression shows how the sound is represented as a sum of echoes of the transmission with associated amplitudes and delays. A time dependency has been introduced in the amplitudes and delays to allow the channel to be time varying. The time variations can be caused by many factors including source/ receiver motion or sea-surface changes.

The situation is a bit more complicated because the amplitudes in Eq. (3) are actually complex numbers. This is due, for example, to bottom reflections that introduce a phase shift (e.g., a $\pi / 2$ phase shift). Since this is a constant phase shift over frequency, it does not simply introduce an additional time delay. Therefore, a more careful application of the convolution theorem is required:

$$
p(t)=\sum_{n=1}^{N} \operatorname{Re}\left[A_{n}(t)\right] s\left[t-\tau_{n}(t)\right]-\operatorname{Im}\left[A_{n}(t)\right] s^{+}\left[t-\tau_{n}(t)\right],
$$

where $s^{+}=\mathcal{H}(s)$ is the Hilbert transform of $s(t)$. The Hilbert transform is a $90^{\circ}$ phase shift of $s(t)$ and accounts for the imaginary part of $A_{n}$. An interpretation of Eq. (4) is that any arbitrary phase change is treated as a weighted sum of the original waveform and its $90^{\circ}$ phase-shifted version. The weighting controls the effective phase shift.

One of the goals of this work is to simulate the received field in cases where the receiver and/or sea surface is in 


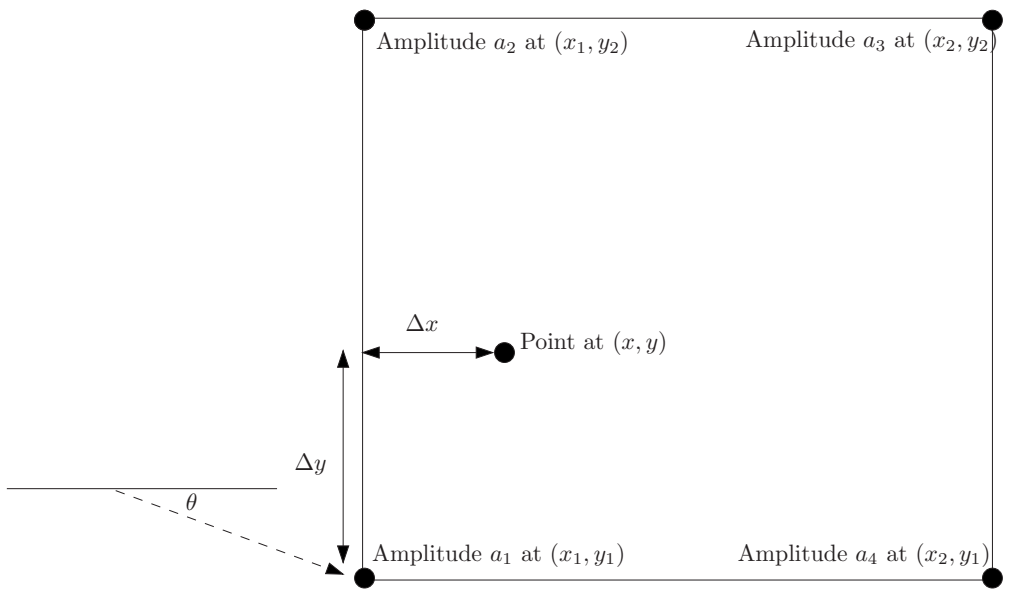

FIG. 4. Four points of the computational grid for a ray trace. The actual arrivals are computed at the four corners and any point in the interior computed through interpolation of the amplitudes and extrapolation of the delays. A sample arrival is shown traveling at angle $\theta$.

motion. In those cases, the arrival amplitudes and delays in Eq. (3) change continuously in time. Therefore, new values for $A_{n}$ and $\tau_{n}$ are required at each time step of the signal transmission. In theory, at each time step, a new set of arrival amplitudes and delays could be computed with an entirely new ray trace from the source to the exact receiver location at that particular time step. However, this would be computationally expensive and mostly unnecessary since the changes (in amplitude and delay) are likely to be very small between time steps. Alternatively, the ray amplitudes and delays are computed on a relatively sparse grid of points in range and depth. The ray information at any given location and time is computed through interpolation. The interpolation scheme is critical to avoid glitches in the final time series that might be caused by jumping too suddenly between points in the computational grid.

The interpolation of ray amplitudes and delays may appear simple enough but there are some subtleties which can cause difficulties. Consider four neighboring grid points as shown in Fig. 4 where at some particular time step, the receiver is located somewhere inside those points. The most straightforward way one might think to calculate the field at this interior point is to identify the same arrival on each of the four corners and then interpolate that arrival amplitude and delay from the four grid points to the receiver location. A problem with that approach occurs when arrival patterns on one grid point do not correspond to those at another. That is, reflections and refraction effects can cause a different number of rays and different ray types on each of the grid points. For example, consider a direct arrival on one corner of the grid that is refracted away from another grid point. In this case, interpolating between these grid points for that arrival number may involve interpolation of a direct path with a bottom bounce path and this will produce incorrect results.

One could keep careful track of all rays and ray types to ensure proper interpolation but that can lead to excessive bookkeeping and storage. Instead, a different interpolation approach is used here. The amplitudes at the four grid points are maintained as separate quantities and their corresponding delays are adjusted by the ray path travel time differences between the corners of the computational grid and the point of the receiver $(x, y)$. The geometry is shown in Fig. 4 with an arrival indicated as a dashed line traveling at angle $\theta$ at the lower left grid point. The delay time for that arrival is adjusted from position $\left(x_{1}, y_{1}\right)$ to position $(x, y)$ by the distance divided by sound speed,

$$
\Delta_{\text {delay }}=(\Delta x \cos \theta+\Delta y \sin \theta) / c,
$$

where, for example, $\Delta x=x-x_{1}$ is positive (increased delay) for position 1 .

The contribution of the arrivals from each of the grid points is weighted according to

$$
\begin{aligned}
& \left(1-w_{1}\right) \times\left(1-w_{2}\right) \times a_{1}, \\
& \left(1-w_{1}\right) \times w_{2} \times a_{2}, \\
& w_{1} \times w_{2} \times a_{3}, \\
& w_{1} \times\left(1-w_{2}\right) \times a_{4},
\end{aligned}
$$

where $a_{1}, a_{2}, a_{3}$, and $a_{4}$ represent the arrival amplitudes at each corner and the weights are

$$
\begin{aligned}
& w_{1}=\left(x-x_{1}\right) /\left(x_{2}-x_{1}\right), \\
& w_{2}=\left(y-y_{1}\right) /\left(y_{2}-y_{1}\right) .
\end{aligned}
$$

Thus, $w_{1}$ represents a proportional distance in the $x$ direction and $w_{2}$ represents a proportional distance in the $y$ direction. To summarize, the received field is constructed using Eq. (4) with an additional sum over each of the four corners (weighted amplitudes).

\section{A. Test cases for source/receiver motion}

The previous section presented the method and here, a few examples are given to illustrate the model at low and high frequencies. The first example will illustrate the quality of the ray interpolation. A tone is transmitted at $350 \mathrm{~Hz}$ from a source at a depth of $30 \mathrm{~m}$ [i.e., continuous wave or (cw)]. The environment has a linearly decreasing sound speed that is $1500 \mathrm{~m} / \mathrm{s}$ at the surface and $1490 \mathrm{~m} / \mathrm{s}$ at the seabed at $100 \mathrm{~m}$ depth. The seabed has a compressional sound speed of $1600 \mathrm{~m} / \mathrm{s}$, density of $1.5 \mathrm{~g} / \mathrm{cm}^{3}$, and attenuation of $0.1 \mathrm{~dB} / \lambda$ (decibels per wavelength). In Fig. 5 is a static frequency domain solution for the transmission loss (TL) out to 


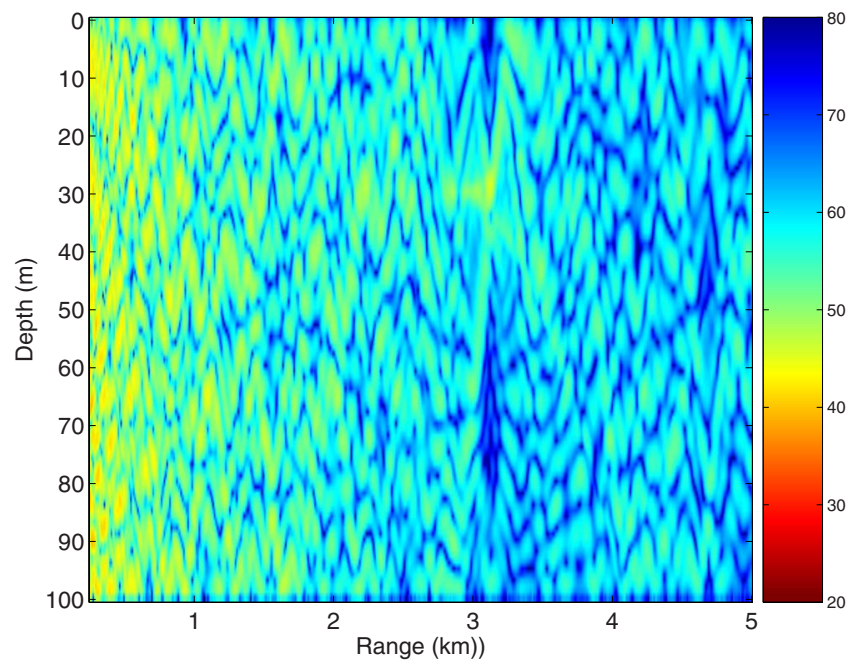

FIG. 5. (Color online) Frequency domain $(350 \mathrm{~Hz})$ TL calculation (dB) over range and depth with the source at a depth of $30 \mathrm{~m}$.

$5 \mathrm{~km}$ in range (i.e., a standard TL calculation). ${ }^{9}$ Next, the time-series simulator records the pressure field on a receiver that sweeps out the same $5 \mathrm{~km}$ range in time. By using 100 of these receivers placed at $1 \mathrm{~m}$ increments in depth, the entire range-depth volume is swept out over $100 \mathrm{~s}$ (each receiver moves from 0 to $5 \mathrm{~km}$ over $100 \mathrm{~s}$ ). The amplitude of these time-series data are plotted on a decibel scale in Fig. 6. For this simulation, a single ray trace computed the arrival amplitudes and delays at each point in a grid of $1 \mathrm{~m}$ in depth and $100 \mathrm{~m}$ in range. Each moving receiver was placed at depths in between these grid points to ensure all computed values were from interpolation rather than exactly falling on grid points. Even for the relatively large grid spacing, the technique produces a good result when compared to the $\mathrm{cw}$ TL in Fig. 5.

The second example is for a higher frequency transmission at $10 \mathrm{kHz}$ and illustrates the interpolation as well as the Doppler effects. The environment is the same as for the first

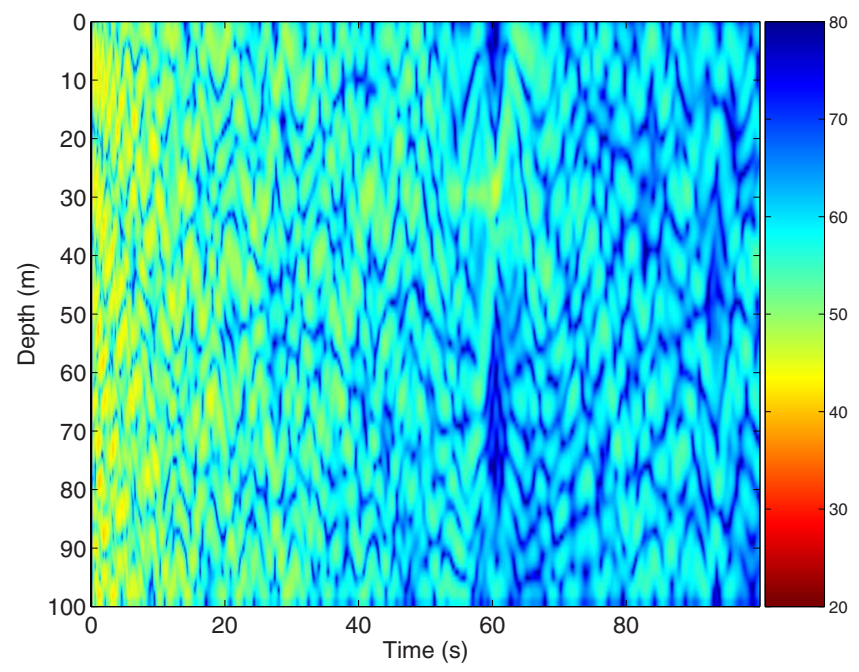

FIG. 6. (Color online) Same as Fig. 5 except calculated using the timedomain approach. That is, a set of moving receivers sweep out (in time) the acoustic TL. Note the different $x$ axes between this figure and Fig. 5. At this low frequency, the Doppler shift is not significant enough to noticeably change the TL.
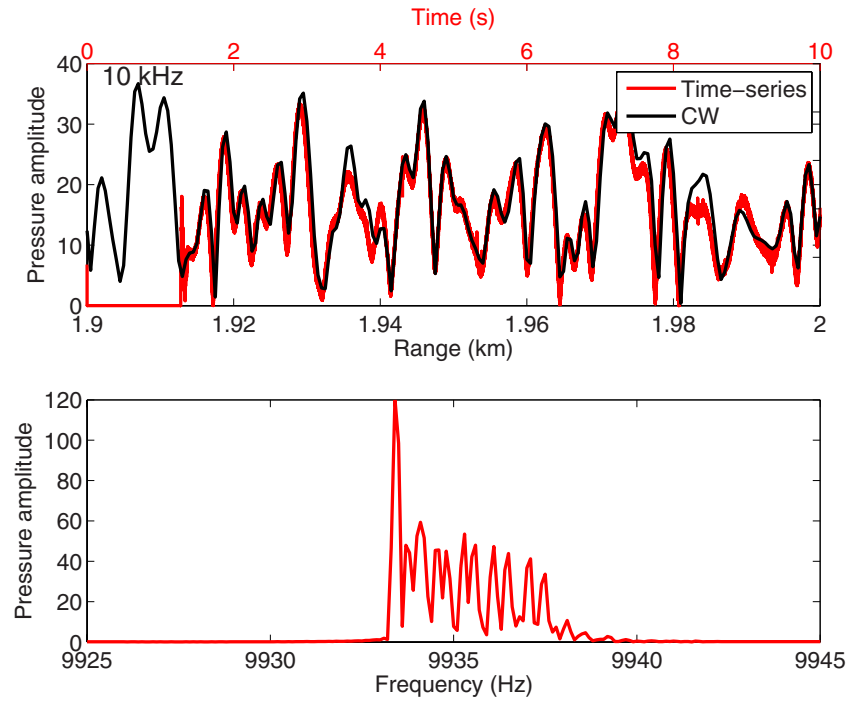

FIG. 7. (Color online) Top panel shows the cw solution vs range and the time-series solution vs time as the receiver moves out in range. The corresponding range and time $x$ axes are indicated. The lower panel shows the spectrum of the time-series solution with the Doppler shifts which differ for the various paths.

example, but a slightly denser grid is used for the ray calculation due to the higher frequency $(0.5 \mathrm{~m}$ depth spacing and $50 \mathrm{~m}$ range spacing). This is still around three wavelengths in depth and several hundred wavelengths in range between grid points. A single line of pressure amplitude is shown in the upper panel of Fig. 7 at a receiver depth of $50.25 \mathrm{~m}$ (between grid points) and is compared to the cw calculation. The moving receiver speed is $10 \mathrm{~m} / \mathrm{s}$ and this introduces a Doppler shift of around $67 \mathrm{~Hz}$ (moving away from the source horizontally). From the agreement in the pressure amplitudes (between static and moving), one might incorrectly assume that the Doppler effects are of little importance. However, various acoustic systems may be significantly impacted by Doppler. For example, the spectrum of the received time series is shown in the lower panel of Fig. 7. The spectrum shows the direct path shifted by the expected $67 \mathrm{~Hz}$ as well as a set of less Doppler-shifted peaks due to the multipath that travels more vertically than the direct path. This type of Doppler spread is one of the primary mechanism that cause channel equalizers to fail in coherent communication schemes (i.e., coherent acoustic modems).

\section{MODELING TIME-VARYING SEA SURFACES}

The previous section only considered the motion of the receiver. This could also approximate the solution for the case when both source and receiver are moving horizontally (possibly at different speeds) in a range independent environment. A time-varying sea surface can be added to the model (with receiver motion only) with slight modifications. In this case, the sea surface is assumed to vary slowly in range as might be the case for swell, ignoring small scale roughness. This limitation will be explored further in the next sections when the rough, time-varying surfaces are considered. To modify the previously described algorithm, additional ray traces are computed to sample the time-evolving sea surface. Figure 8 diagrams the required interpolation scheme to in- 


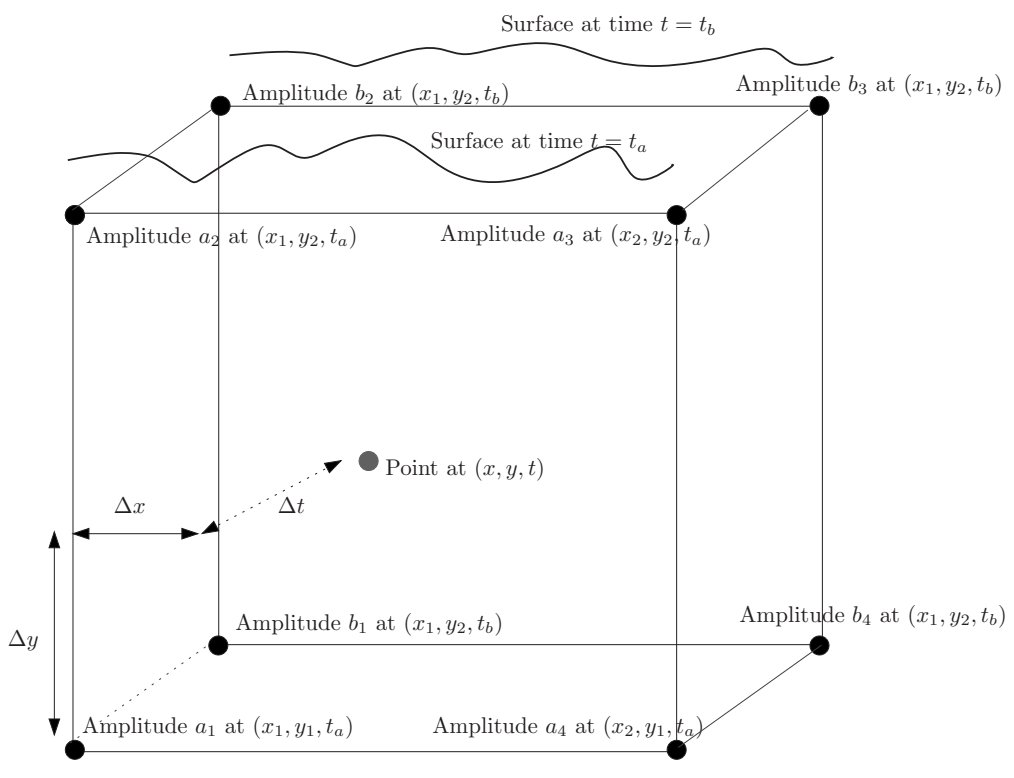

FIG. 8. Same as Fig. 4 except with the added dimension to allow for time changing surface.

clude surface motion. At an initial time, $t=t_{a}$, the surface can have an arbitrary shape and this evolves in time to produce a surface shape defined at time $t=t_{b}$. The time interval to sample the surface adequately is on the order of the time interval of the swell being modeled. This is typically much less than the sampling interval for the acoustic transmission. Therefore, ray arrival amplitudes and delays are computed for surfaces at $t=t_{a}$ and $t=t_{b}$ and trilinear interpolation is used in much the same way as described previously with bilinear interpolation. One difference is that the delays are not extrapolated for this third dimension. The proper arrival amplitudes and delays are simply determined through the weights applied to the eight corners of the computation grid. As with the previous two dimensions, the arrivals are kept as separate quantities on each of the eight corners of the cube depicted in Fig. 8. The delays at each corner of the cube are advanced or retarded according to distance and the weight given to each corner is determined by

$$
\begin{aligned}
& \left(1-w_{1}\right) \times\left(1-w_{2}\right) \times\left(1-w_{3}\right) \times a_{1}, \\
& \left(1-w_{1}\right) \times w_{2} \times\left(1-w_{3}\right) \times a_{2}, \\
& w_{1} \times w_{2} \times\left(1-w_{3}\right) \times a_{3}, \\
& w_{1} \times\left(1-w_{2}\right) \times\left(1-w_{3}\right) \times a_{4}, \\
& \left(1-w_{1}\right) \times\left(1-w_{2}\right) \times w_{3} \times b_{1}, \\
& \left(1-w_{1}\right) \times w_{2} \times w_{3} \times b_{2}, \\
& w_{1} \times w_{2} \times w_{3} \times b_{3}, \\
& w_{1} \times\left(1-w_{2}\right) \times w_{3} \times b_{4},
\end{aligned}
$$

where $a_{1}, a_{2}, a_{3}$, and $a_{4}$ represent the arrival amplitudes at each corner at $t=t_{a}$ and $b_{1}, b_{2}, b_{3}$, and $b_{4}$ represent the arrival amplitudes at each corner at $t=t_{b}$. The weights are

$$
w_{1}=\left(x-x_{1}\right) /\left(x_{2}-x_{1}\right),
$$

$$
\begin{aligned}
& w_{2}=\left(y-y_{1}\right) /\left(y_{2}-y_{1}\right), \\
& w_{3}=\left(t-t_{a}\right) /\left(t_{b}-t_{a}\right) .
\end{aligned}
$$

The time steps, $t_{a}$ and $t_{b}$, where the ray traces are computed, are typically at time intervals much greater than the acoustic sampling time interval. Therefore, many of the time steps rely on interpolated arrival information.

\section{A. Test cases for source/receiver motion with a time- varying sea surface}

To check the developed algorithm, a comparison can be made between results using the approach outlined in the previous sections with an exact solution. The exact solution requires the water column to be isospeed and the sea surface to be flat but can vary in height over time (i.e., a flat surface that can move up and down). This is a reasonable comparison since the approach outlined using a ray tracing algorithm is the same whether the sound speed is depth dependent (or not) and regardless of the shape of the surface.

The exact solution is developed based on the method of images $^{9}$ and the geometry is given in Fig. 9. The pressure field is constructed using Eq. (4) with a set of amplitudes $A_{m n}$ and corresponding delays $\tau_{m n}$ with $n=1,2,3,4$ and $m=0$ $\rightarrow \infty$. The amplitudes and delays are as follows:

$$
\begin{aligned}
A_{m n} & =\frac{1}{R_{m n}}, \\
R_{m n} & =\sqrt{r^{2}+z_{m n}^{2}}, \\
\tau_{m n} & =\frac{R_{m n}}{c}, \\
z_{m 1} & =2 D m z_{s}+z_{r}, \\
z_{m 2} & =2 D(m+1)-z_{s}-z_{r}, \\
z_{m 3} & =2 D m+z_{s}+z_{r},
\end{aligned}
$$




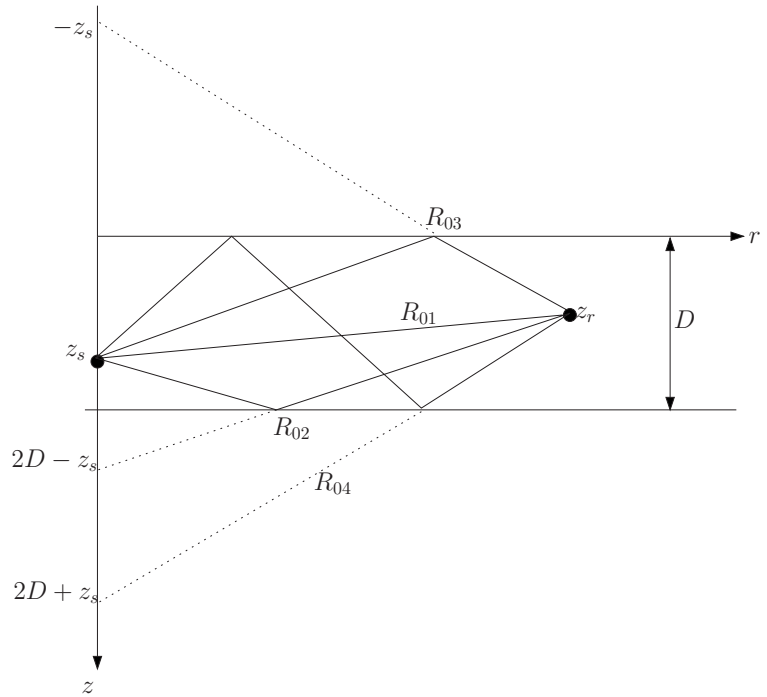

FIG. 9. Geometry showing the location and ranges of the source, receiver, and corresponding images.

$$
z_{m 4}=2 D(m+1)+z_{s}-z_{r},
$$

where $z_{s}$ is the source depth, $z_{r}$ is the receiver depth, $r$ is the receiver range, $D$ is the water depth, and $c$ is the water sound speed (isospeed at $1500 \mathrm{~m} / \mathrm{s}$ ). The one twist in this standard formulation is that the surface is moving in time, which causes the amplitudes and delays to vary in time. The variations in the surface are incorporated into the terms of Eq. (10) through adding a time dependence to $D, z_{s}$, and $z_{r}$ since the effective water depth and the source and receiver depths change with time. By making use of Eq. (4) with these timedependent amplitudes and delays, the exact received time series can be determined.

To illustrate the comparison, consider an example with $D=100 \mathrm{~m}, z_{s}=30 \mathrm{~m}, z_{r}=40 \mathrm{~m}$, and $r=250 \mathrm{~m}$. The receiver is moving toward the source at $0.75 \mathrm{~m} / \mathrm{s}$. The sea surface is moving away from the seabed at a speed of $0.25 \mathrm{~m} / \mathrm{s}$. In this way, the Doppler shifts for surface interacting paths will be reduced from $0.75 \mathrm{~m} / \mathrm{s}$. The waveform chosen for this example is extremely Doppler sensitive such that the multipath (each having distinct Doppler shifts) can be separated in both delay time and Doppler space. The transmission waveform used is the BPSK signal that was used in the Makai experiment. The separation between grid points (as in the cube shown in Fig. 8) are as follows: the depth spacing was $1 \mathrm{~m}$, the range spacing was $5 \mathrm{~m}$, and the distance between surfaces was $0.0125 \mathrm{~m}$; this implies a new surface every $12.5 \mathrm{~ms}$ (i.e., 40 ray traces were computed for surface perturbations between 0 and $0.5 \mathrm{~m}$ ).

The results are shown in Fig. 10. In panel (a) of the figure is the exact impulse response shown in delay and Doppler space (i.e., the "scattering function"). In panel (b) of Fig. 10 is the arrival structure using the time-dependent ray interpolation algorithm previously described. In (a) and (b), the first path is the direct one and has a Doppler shift corresponding to $-0.75 \mathrm{~m} / \mathrm{s}$ due to the horizontal receiver motion (directly toward the source). The next path is due to the surface bounce and is less Doppler shifted due to the seasurface motion acting to Doppler shift in the opposite direc-
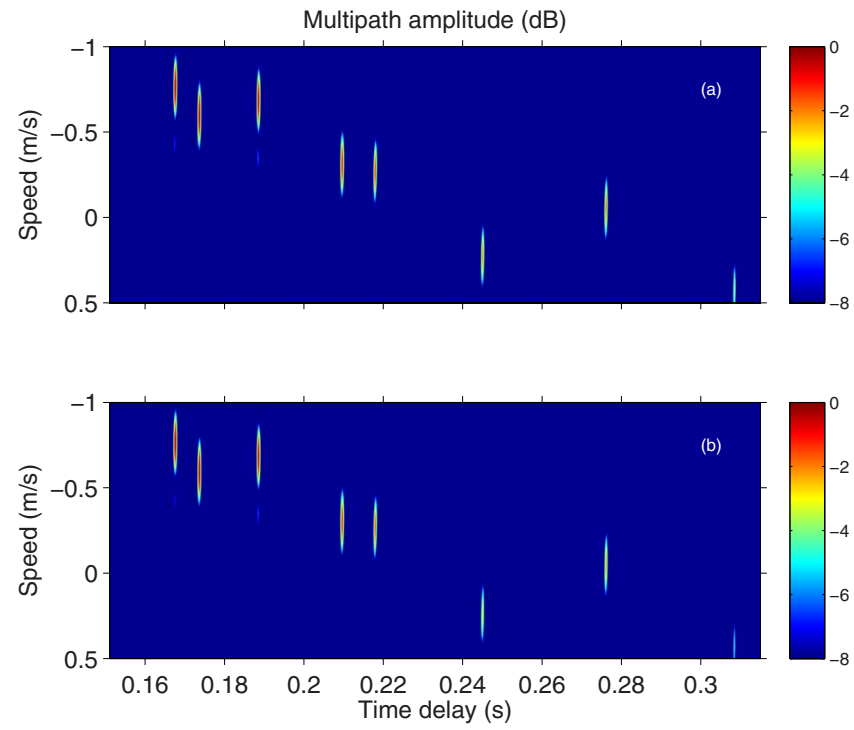

FIG. 10. (Color online) In panel (a), the exact impulse response (normalized) is computed using the method of images and in (b), it is computed using the time-dependent ray interpolation method described. The first arrival in time is from the direct path and shows only a Doppler shift due to the receiver horizontal motion of $-0.75 \mathrm{~m} / \mathrm{s}$. The next two paths that follow are from the surface and bottom bounces. The additional multipaths are due to multiple interactions with the sea surface and the bottom.

tion. The next path is the bottom bounce and this path is unaffected by the surface motion and is Doppler shifted less than the direct path due to the more vertical directionality of the ray path. The next arrivals are due to multiple bottomsurface bounces.

\section{MODELING STATIC, ROUGH SEA SURFACES}

The previous section developed a method for modeling time series with gently varying sea surfaces like that from swell and the next sections provide an alternative formulation which is more appropriate for finer scale roughness. Rough surface scattering has been extensively studied and there is a huge amount of literature on the subject (see, for example, the text by Ogilvy). ${ }^{12}$ To begin, exact solutions for one-dimensional static rough surfaces are described by using the Helmholtz integral equation. This will be compared to solutions found with a ray trace solution (e.g., Bellhop) with surface roughness. This will be further developed with the use of the Kirchhoff approximation to yield a time-domain version for scattering from rough, time-evolving surfaces.

\section{A. Helmholtz integral equation}

The description in this section will follow the notation and derivation from Thorsos. ${ }^{13}$ Those results are presented for completeness and to establish the notation being used. The exact solution to the time-harmonic $\left(e^{-i \omega t}\right)$ onedimensional sea surface (free surface boundary) is given by the Helmholtz integral equation,

$$
p(\bar{r})=p_{\text {inc }}(\bar{r})-\frac{1}{4 i} \int_{S} H_{0}^{(1)}\left(k\left|\bar{r}-\bar{r}^{\prime}\right|\right) \frac{\partial p\left(\bar{r}^{\prime}\right)}{\partial n^{\prime}} d s^{\prime} .
$$

That is, the total field is a sum of the incident and scattered fields, $p(\vec{r})=p_{\text {inc }}(\vec{r})+p_{\text {scat }}(\vec{r})$. In Eq. (11), the acoustic wave 


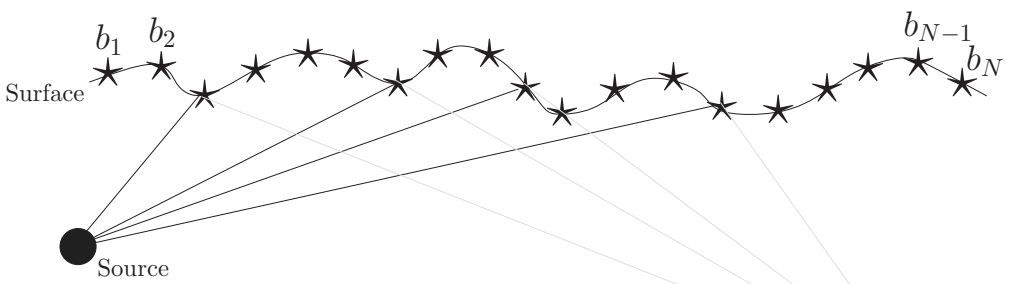

FIG. 11. Diagram illustrating the surface is replaced by a set of Huygens sources.

number is given by $k=\omega / c$, the quantity $\partial p\left(\bar{r}^{\prime}\right) / \partial n^{\prime}$ is the normal derivative of the pressure field on the surface, and $H_{0}^{(1)}$ is the zero-order Hankel function of the first kind. On the surface the pressure field is zero, such that

$$
p_{\text {inc }}(\bar{r})=\frac{1}{4 i} \int_{S} H_{0}^{(1)}\left(k\left|\bar{r}-\bar{r}^{\prime}\right|\right) \frac{\partial p\left(\bar{r}^{\prime}\right)}{\partial n^{\prime}} d s^{\prime} .
$$

The quantity of interest is the total pressure field $p(\vec{r})$ which requires first solving Eq. (12) for $\partial p\left(\bar{r}^{\prime}\right) / \partial n^{\prime}$.

Equation (12) can be approximately solved by using numerical integration,

$$
a_{m}=\sum_{n=1}^{N} A_{m n} b_{n}, \quad m=1, \ldots, N,
$$

where $a_{m}$ is the incident field, and $A_{m n}$ are the Hankel functions,

$$
\begin{aligned}
& a_{m}=p_{\text {inc }}\left(\bar{r}_{m}\right), \\
& A_{m n}= \begin{cases}H_{0}^{(1)}\left(k \mid \bar{r}_{m}-\bar{r}_{n}\right) & \text { if } m \neq n \\
H_{0}^{(1)}\left[(k \Delta x / 2 e) \gamma_{m}\right] & \text { if } m=n,\end{cases}
\end{aligned}
$$

and

$$
b_{n}=\left.\frac{\Delta x}{4 i} \gamma_{n} \frac{\partial p\left(\bar{r}^{\prime}\right)}{\partial n^{\prime}}\right|^{\bar{r}_{n}} .
$$

The surface height function is defined as $f(x)$ with $\gamma^{2}\left(x^{\prime}\right)$ $=1+\left[d f\left(x^{\prime}\right) / d x^{\prime}\right]^{2}$ and $d s^{\prime}=\gamma\left(x^{\prime}\right) d x^{\prime}$. Using unit vectors $\hat{x}$ and $\hat{z}$, the vector $\bar{r}$ is defined as $\bar{r}_{m}=x_{m} \hat{x}+f\left(x_{m}\right) \hat{z}$ and $x_{m}$ $=(m-1) \Delta x-L / 2$, with $\gamma_{m}=\gamma\left(x_{m}\right)$ and $L$ being the total length of the surface.

By using matrix notation, Eq. (13) can be written as

$$
\mathbf{a}=\mathbf{A b} \text {, }
$$

with solution for $\mathbf{b}$ determined through inversion of $\mathbf{A}$,

$$
\mathbf{b}=\mathbf{A}^{-1} \mathbf{a} \text {. }
$$

Once this equation is solved for $\mathbf{b}$, the scattered field is obtained by using

$$
p_{\text {scat }}(\vec{r})=\sum_{n=1}^{N} H_{0}^{(1)}\left(k\left|\bar{r}_{m}-\bar{r}_{n}\right|\right) b_{n} .
$$

The practical limitations of numerically solving these equations is the inversion of matrix A. In practice, the sampling of the surface requires approximately five points per wavelength. To keep from introducing artifacts, the computational domain has to be even larger than the region of in- terest (here, it was extended by $250 \mathrm{~m}$ ). If the objective is to solve a problem with a $1 \mathrm{~km}$ surface at $10 \mathrm{kHz}$ (for example, for an acoustic communication simulation), the number of discrete points is over 30000 , which requires inversion of a $30000 \times 30000$ matrix for each frequency. This increases to $300000 \times 300000$ for a $10 \mathrm{~km}$ simulation. Add to this the requirement that this be done over a broad band of frequencies for many practical problems, and it becomes even more difficult. To make matters worse, if the objective is to determine the time-evolving nature of the scattering, this must be done at many time steps. The size of the problem by using exact solutions becomes apparent and leads to the approximations used in the next sections. The exact approach outlined here is valuable, however, to provide ground-truth comparison with the approximate methods.

\section{B. Ray approach with the Kirchhoff approximation}

The Kirchhoff approximation is as follows:

$$
\frac{\partial p(\bar{r})}{\partial n} \cong 2 \frac{\partial p_{\text {inc }}(\bar{r})}{\partial n} \text {. }
$$

The value of this approximation is that the elements of $b_{n}$ required for the scattered field are no longer dependent on the total field but only on the incident field. This removes the need to store and invert the matrix A. Another way to think about the solution for the scattered field is that the surface is removed and replaced by point sources at each discrete location $x_{n}$. The weight of each point source is determined by the coefficients $b_{n}$. This is basically a statement of the Huygens principle ${ }^{14}$ and is depicted in Fig. 11. For the exact solution, each point source amplitude $b_{n}$ depends on each of the others, while for the Kirchhoff approximation, it is only a local reflection. However, the Huygens sources can reradiate in all directions and the dense sampling allows for a more complicated scattered field than that from a specularly reflected ray trace.

The incident field can be written in terms of a ray amplitude and delay (far field approximation for the Hankel Function) from the source to each of the Huygens secondary sources,

$$
p_{\text {inc }}\left(\bar{r}_{n}\right) \cong \frac{1}{\sqrt{k R_{1 n}}} e^{i \omega \tau_{1 n}},
$$

where $R_{1 n}$ and $\tau_{1 n}$ are computed similarly to Eq. (10) for the 01 path for the surface bounce (suppressing the first index which is unnecessary here since there are no higher order images). Since the Huygens reconstruction removes the sur-

M. Siderius and M. B. Porter: Modeling in time-varying channels 
face boundary and replaces it with secondary sources, the only other path to arrive in the incident field is from the bottom bounce path 02 . The total incident field at each Huygens secondary source has two terms,

$$
p_{\text {inc }}\left(\bar{r}_{n}\right)=\frac{e^{i \omega \tau_{1 n}}}{\sqrt{k R_{1 n}}}+\frac{V_{n} e^{i \omega \tau_{2 n}}}{\sqrt{k R_{2 n}}},
$$

where the second term has been modified to include the bottom loss $V_{n}$ that occurs for a nonpressure release bottom. This term will generally be a function of the angle incident on the bottom and is therefore indexed to each point on the surface. Further, this assumes a plane wave reflection loss which is a slight approximation. The expression for the incident field has been written out for this isospeed case for clarity; however, these two terms can be easily taken from a ray trace algorithm that would include refraction for more realistic cases. The weights for the Huygens secondary sources are computed using Eq. (15) and taking the derivative of $p_{\text {inc }}\left(\bar{r}_{n}\right)$,

$$
b_{n} \cong k \tilde{N}_{n}\left(\frac{e^{i \omega \tau_{1 n}}}{\sqrt{k R_{1 n}}}+\frac{V_{n} e^{i \omega \tau_{2 n}}}{\sqrt{k R_{2 n}}}\right),
$$

where

$$
\tilde{N}_{n}=\frac{e^{-i \pi / 4} \Delta x \gamma_{n}}{\sqrt{2 \pi}}\left(\hat{s}_{r, n} \cos \theta_{n}+\hat{s}_{z, n} \sin \theta_{n}\right),
$$

with $\hat{s}_{r, n} \hat{s}_{z, n}$ the unit vector components for the normal to the surface at $r_{n}$ and $\theta$ the direction of propagation of $p_{\text {inc. }}$. The $\tilde{N}$ and $k$ in Eq. (22) appear due to the derivative in the direction normal to the surface. The value of $b_{n}$ is an approximation to the derivative since the higher order terms of the derivative are neglected (i.e., the terms with $R^{-3 / 2}$ ).

For the general cases considered here, there is a seabed boundary so the free-space Hankel function is replaced in Eq. (11) with the Green's function $G\left(k\left|\bar{r}-\bar{r}^{\prime}\right|\right)$ representing the point source response between $\bar{r}$ and $\bar{r}^{\prime}$.

$$
p(\bar{r})=p_{\text {inc }}(\bar{r})-\frac{1}{4 i} \int_{S} G\left(k\left|\bar{r}-\bar{r}^{\prime}\right|\right) \frac{\partial p\left(\bar{r}^{\prime}\right)}{\partial n^{\prime}} d s^{\prime} .
$$

For the numerical implementation, the Green's function has a similar form as the incident field,

$$
G\left(\left|\bar{r}-\bar{r}_{n}\right|\right)=\frac{e^{i \omega \bar{r}_{1 n}}}{\sqrt{k \widetilde{R}_{1 n}}}+\frac{V_{n} e^{i \omega \bar{\tau}_{2 n}}}{\sqrt{k \widetilde{R}_{2 n}}},
$$

where in this case, $\widetilde{R}_{1 n}$ represents the distance for the direct path between the Huygens secondary source (at $r_{n}$ ) and the field point (at $\bar{r}$ ) and $\widetilde{R}_{2 n}$, represents the distance for the bottom bounce path.

The scattered field is then constructed as

$$
p_{\text {scat }}(\bar{r})=\sum_{n=1}^{N} G\left(k\left|\bar{r}-\bar{r}_{n}\right|\right) b_{n},
$$

where the total scattered field is a sum of four components for each Huygens source,

$$
p_{\text {scat }}(\bar{r})=\sum_{n=1}^{N} \sum_{m=1}^{4} \tilde{N}_{n}\left\{A_{m} e^{i \omega T_{m}}\right\},
$$

with the amplitudes

$$
\begin{aligned}
& A_{1}=\frac{1}{\widetilde{R}_{1 n} R_{1 n}}, \\
& A_{2}=\frac{V_{n}}{\widetilde{R}_{2 n} R_{1 n}}, \\
& A_{3}=\frac{V_{n}}{\widetilde{R}_{1 n} R_{2 n}}, \\
& A_{4}=\frac{V_{n}^{2}}{\widetilde{R}_{2 n} R_{2 n}},
\end{aligned}
$$

and the delays

$$
\begin{aligned}
& T_{1}=\widetilde{\tau}_{1 n}+\tau_{1 n}, \\
& T_{2}=\widetilde{\tau}_{2 n}+\tau_{1 n}, \\
& T_{3}=\widetilde{\tau}_{1 n}+\tau_{2 n}, \\
& T_{4}=\widetilde{\tau}_{2 n}+\tau_{2 n} .
\end{aligned}
$$

The frequency dependent $k$ term conveniently cancels out. However, this is a line source formulation and this frequency dependence will become necessary when extending to a point source, which is required for typical applications. This issue will be discussed further in Sec. VI. It is also worth noting that this formulation includes one surface bounce path. That is, the total field will consist of a direct path, bottom-bounce, bottom-surface-bounce, surface-bottombounce, and bottom-surface-bottom-bounce paths. For many applications, these six paths will provide a sufficient impulse response representation. However, paths with multiple surface bounces could be included in an approximate way by modifying the amplitude of the first surface bounce by taking only the specular path and then include the scattering in the second interaction with the surface.

\section{Examples of scattering approaches for static surfaces}

Static surfaces at a single frequency are useful for checking the approximate solutions since the exact Helmholtz-Kirchhoff integral equation can be used as the ground truth. The time-domain Kirchhoff approach will not immediately show its utility for these time-harmonic examples. However, that formulation will be critical when extending to the time-varying surfaces in Sec. VII.

\section{Surface scattering without a seabed}

In the first example, the field interactions with the rough sea surface is determined without a seabed. Using an isospeed water column allows the exact Hankel functions to be 

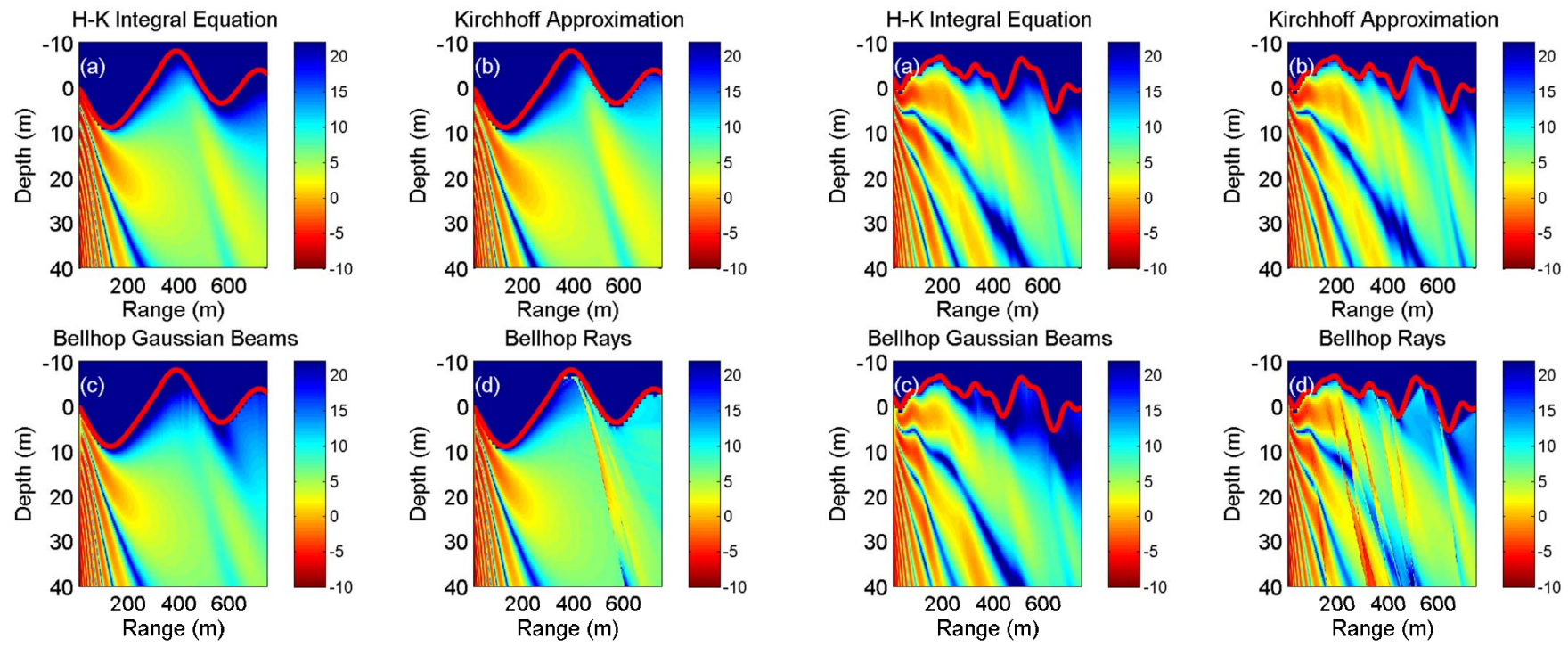

FIG. 12. (Color online) TL (referenced to $1 \mu \mathrm{Pa}$ at $1 \mathrm{~m}$ ) for different numerical methods with a rough surface and no seabed. The source depth was $40 \mathrm{~m}$ from the surface and the frequency was $200 \mathrm{~Hz}$, the surface height standard deviation is $0.5 \lambda$ and the correlation length is $50 \lambda$. Panel (a) is the exact solution using the Helmholtz-Kirchhoff integral equation, in (b) the solution shown is for the time-domain Kirchhoff approximation and in (c) is the output from the Bellhop ray/beam trace using Gaussian beams and (d) for geometric rays.

included in the Helmholtz-Kirchhoff integral equation. The source depth was $40 \mathrm{~m}$ from the surface and the frequency was $200 \mathrm{~Hz}$.

To generate the random surface shape, a spectral method was used similar to that by Thorsos. ${ }^{13}$ That is, white noise is filtered with a Gaussian spectrum to provide a onedimensional random sequence with height and correlation distance governed by the parameters of the Gaussian density, its mean, and correlation. For the first example, the surface height standard deviation is $0.5 \lambda$ and the correlation length is $50 \lambda$.

The results are shown in the four panels in Fig. 12. The field is displayed as TL (TL is referenced to $1 \mu \mathrm{Pa}$ at $1 \mathrm{~m}$ ) with the line near depth 0 being the shape of the sea surface. Panel (a) is the exact solution using the Helmholtz-Kirchhoff integral equation. In (b), the solution shown is for the timedomain Kirchhoff approximation. In (c) is the output from the Bellhop ray/beam trace using Gaussian beams and (d) is the same using geometric rays. The results show nearly perfect agreement between (a), (b), and (c) while the geometric rays in (d) show indications of typical ray artifacts. These types of artifacts are the motivation for improved methods such as Gaussian beams.

In Fig. 13, the correlation length is reduced to $20 \lambda$. In this case, the geometric rays in panel (d) are clearly showing artifacts while the Kirchhoff method (b) is still in reasonable agreement with the exact solution in (a) and to some degree also the Gaussian beam solution in (c). Although these are just samples, the results for other cases were similar. That is, as the roughness increased (either by reducing the correlation length or the height) the geometric beams appeared to produce erroneous results first, followed by the Gaussian beams, and then the Kirchhoff approach.

FIG. 13. (Color online) Same as Fig. 12 except with surface roughness correlation is $20 \lambda$.

\section{Surface scattering with a seabed}

As a second example, the seabed was included in the calculation. As before, the surface had $20 \lambda$ correlation length, surface height standard deviation of $0.5 \lambda$, and the source was at $40 \mathrm{~m}$ depth. The water column was $100 \mathrm{~m}$ with a homogeneous (in depth) seabed with a sound speed of $1600 \mathrm{~m} / \mathrm{s}$, density of $1.5 \mathrm{~g} / \mathrm{cm}^{3}$, and attenuation factor of $0.75 \mathrm{~dB} / \lambda$. Since the Kirchhoff approximation method includes only a single surface bounce path, the figure that it is compared against was computed with the same paths. In Fig. 14 , results from a single realization are shown comparing the Helmholtz-Kirchhoff integral equation result with the Kirchhoff approximation.

The results shown are for just single realizations of the random rough surface; however, these were typical and showing results for many realizations is not possible here. Results for many more realizations are summarized by the plots in Fig. 15. In this figure, there are three panels showing TL at 200, 400, and $600 \mathrm{~Hz}$ for the same conditions as the previous case (i.e., source depth of $40 \mathrm{~m}$ and $100 \mathrm{~m}$ water column). In each panel, 50 realizations of rough surfaces are averaged. This is similar to the comparisons made by Thorsos ${ }^{13}$ but those were for a scattering cross section and here the results are for a receiver at a fixed depth of $25 \mathrm{~m}$.
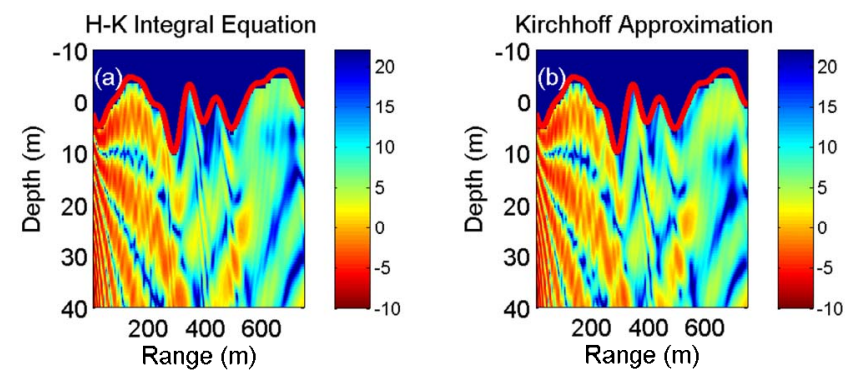

FIG. 14. (Color online) Same as Fig. 13 except with the inclusion of a smooth seabed at $100 \mathrm{~m}$ depth. Panel (a) is the exact solution using the Helmholtz-Kirchhoff integral equation. In (b), the solution shown is for the time-domain Kirchhoff approximation. 

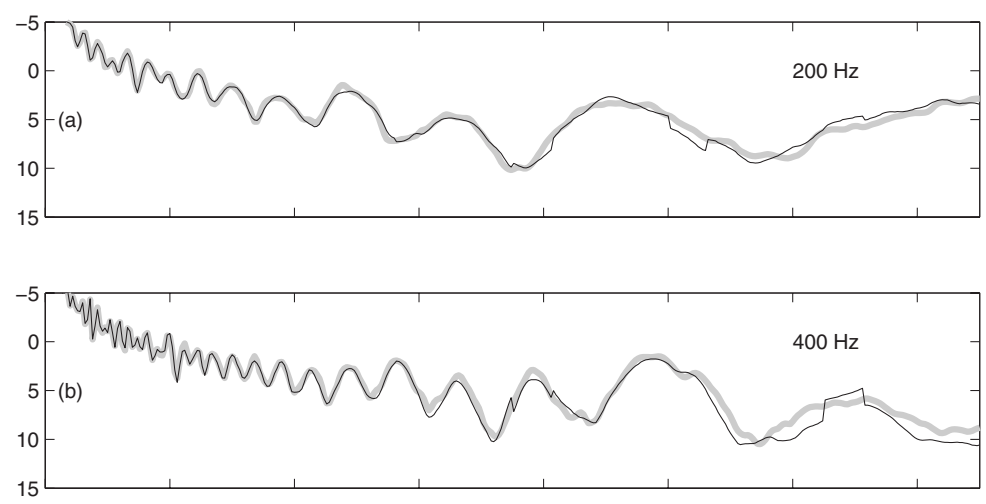

FIG. 15. Averaged TL for 50 realizations at frequencies of $200 \mathrm{~Hz}$ in (a), $400 \mathrm{~Hz}$ in (b), and $600 \mathrm{~Hz}$ in (c). The heavy gray line is the Helmholtz-Kirchhoff result and the thin black line is the Kirchhoff approximation.

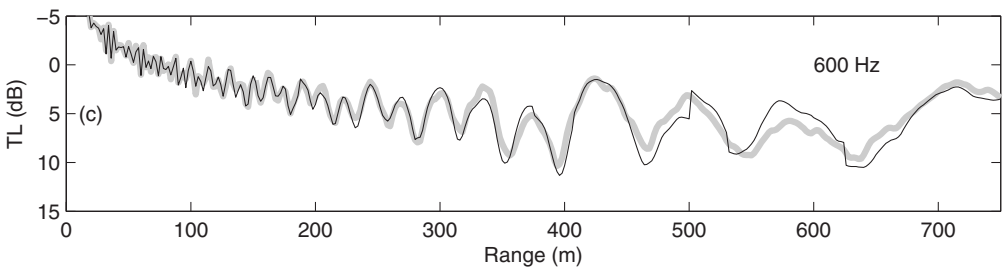

The heavier gray line indicates the Helmholtz-Kirchhoff integral equation results and the thin black line is for the Kirchhoff approximation. In general, not only the average levels are in good agreement, but also the details of the finer structure.

\section{APPROXIMATING POINT SOURCE SOLUTIONS FROM LINE SOURCES}

The previous solutions using the Helmholtz-Kirchhoff integral equation as well the Kirchhoff approximation assumed line sources for the excitation field. This is convenient for the derivations but is not particularly good for simulating the types of sound sources used in underwater acoustic systems. These are much more accurately modeled using point sources. The line source solutions can be converted to point source solutions in a fairly simple way.

Consider the field from a line source in free space,

$$
p_{L}(R)=\frac{i}{4} H_{0}^{(1)}(k R) \simeq \sqrt{\frac{i}{8 \pi k R}} e^{i k R}
$$

where, as before, $k$ is the acoustic wave number and $R=\mid \bar{r}$ $-\bar{r}^{\prime} \mid$ is the distance between the source and receiver. The point source field is

$$
p_{P}(R)=\frac{e^{i k R}}{4 \pi R} .
$$

The line source field is converted to an equivalent point source field by multiplying by the factor $\sqrt{k / i 2 \pi R}$,

$$
p_{P}(R)=\sqrt{\frac{k}{i 2 \pi R}} p_{L}(R) .
$$

An additional complication arises since the line source solution is determined in an $x-z$ plane geometry coordinate system. Therefore, the true range $R$ from the source to receiver is related to the plane geometry distance $x$ according to $x$ $=R \cos \alpha$, where $\alpha$ is the angle from the horizontal line between the source and the receiver. The conversion from a plane-geometry line source solution to an equivalent axisymmetric, cylindrical geometry, point source solution is then

$$
p_{P}(r, z)=\sqrt{\frac{k \cos \alpha}{i 2 \pi|x|}} p_{L}(x, z),
$$

where, for the cylindrical coordinate system, $r=|x|$. The $\sqrt{\cos \alpha}$ term is generally small except at short ranges where there can be strong arrivals propagating at steep angles. The main amplitude contribution to the conversion factor is from the $\sqrt{x}$ term in the denominator. It will be seen in the next section that the $\sqrt{k}$ term is also important in forming the time-domain solution.

As an example of the correction from a line source solution to a point source solution, the exact field was computed for a point source using two reflecting boundaries separated by $100 \mathrm{~m}$ and the point source at $40 \mathrm{~m}$ depth. This was computed using the method of images. This result is compared to the described Kirchhoff approximation for a line source with the conversion factor applied. Since the boundaries are flat, the Kirchhoff approximation is exact for the line source. Note that the $\sqrt{\cos \alpha}$ term is applied to just the incident field in Eq. (21) according to the source launch angle. The full solution includes an infinite number of reflections. However, as previously mentioned, the Kirchhoff formulation only includes paths with a single surface reflection so the image solution is computed and limited to the same number of paths. In Fig. 16, the exact solution and the line source converted solution are shown for three receiver depths. The lines are nearly on top of each other so the exact solution is shown as a slightly thicker light gray line and the converted line source result is in black.

The conversion of line source results to an equivalent point source result comes about in various propagation solutions. For example, the Gaussian beam formulation also has this same factor which is derived more formally by Porter and Bucker. ${ }^{11}$ Another version of this conversion can be il- 

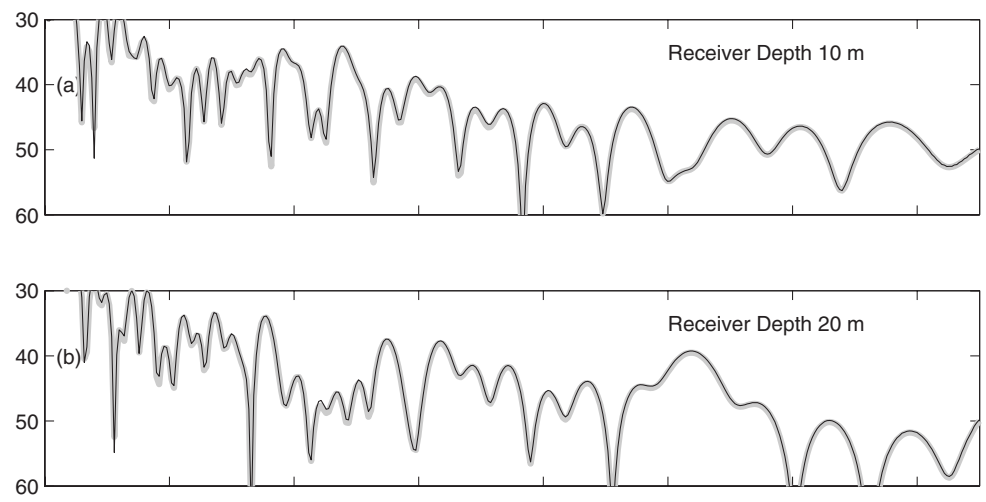

FIG. 16. TL at $200 \mathrm{~Hz}$ for an exact (light gray line) point source and a conversion factor applied to the line source solution (black line). The three panels are for different receiver depths, $10 \mathrm{~m}$ in (a), $20 \mathrm{~m}$ in (b), and $30 \mathrm{~m}$ in (c) for a source at $40 \mathrm{~m}$.

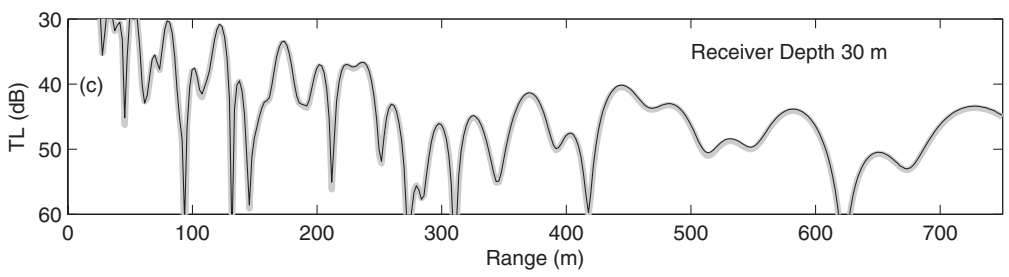

lustrated for waveguide propagation using normal modes. The pressure field for a line source is derived by Jensen et $a{ }^{9}{ }^{9}$ as

$$
p_{L}(x, z)=\sum_{m=1}^{\infty} \frac{i}{2 \rho\left(z_{s}\right)} \Psi_{m}\left(z_{s}\right) \Psi_{m}(z) \frac{e^{i k_{m}|x|}}{k_{m}},
$$

where the depth dependent mode functions are $\Psi$, the source depth is $z_{s}$, the receiver depth is $z$, the density is $\rho$, and the horizontal wave number that corresponds to the mode is $k_{m}$. The corresponding modal sum for the axial symmetric point source in cylindrical coordinates $(r, z)$ is

$$
p_{P}(r, z)=\sum_{m=1}^{\infty} \frac{1}{\rho\left(z_{s}\right)} \sqrt{\frac{i}{8 \pi r}} \Psi_{m}\left(z_{s}\right) \Psi_{m}(z) \frac{e^{i k_{m} r}}{\sqrt{k_{m}}},
$$

where $|x|=r$. To obtain the point source solution from the line source solution requires multiplying $p_{L}(x, z)$ by $\sqrt{k_{m} / i 2 \pi|x|}$, that is,

$$
p_{P}(r, z)=\sqrt{\frac{k_{m}}{i 2 \pi|x|}} p_{L}(x, z) .
$$

Representing the horizontal wave numbers in terms of the corresponding angle, $k_{m}=k \cos \alpha$, results in the conversion factor $\sqrt{k \cos \alpha / i 2 \pi|x|}$, which is exactly the same as shown previously for free space. The weighting of the rays by $\sqrt{\cos \alpha}$ according to their launch angle would be equivalent to weighting the mode function source excitation by this factor, that is, $\Psi_{m}\left(z_{s}\right) \sqrt{\cos \alpha}$. As mentioned, the greatest influence is due to the $\sqrt{|x|}$ term in the denominator and, for the time-domain solution, the $\sqrt{k}$ factor.

\section{TIME-DOMAIN KIRCHHOFF APPROXIMATION FOR TIME-VARYING SURFACES}

The approach outlined in Sec. V with the Kirchhoff approximation can be applied to time-varying sea surfaces. The surface shape varies at each time step and the Kirchhoff solution is recomputed. This is possible since the Kirchhoff approximation is numerically efficient and does not require the matrix inversion needed for the integral equation. There is one subtle point to obtain a time series that resembles measurements. In Sec. VI, it was pointed out that to obtain the field from a point source, the line source solution required multiplying by $\sqrt{k \cos \alpha / i 2 \pi|x|}$. The $\sqrt{1 /|x|}$ term is easy enough to include at each receiver position after the simulation. The $\sqrt{\cos \alpha}$ factor is applied to each of the rays launched from the source according to their angle. The $\sqrt{k}$ factor depends on each frequency component but is also simple to include through the source function. The Fourier transform of the transmit waveform, $S(\omega)$, is multiplied by the $\sqrt{k}$ factor and inverse Fourier transformed back to the time domain as the new transmit waveform used in the convolution sum.

Three examples will be given using this approach. In the first case a flat sea surface moves uniformly. The second example uses a rough, moving sea surface at a single point at a relatively high frequency. The third case is a lower frequency example showing a pulse propagating and reflecting off the rough surface.

\section{A. Modeling a flat, time-varying surface}

This example for a flat but moving surface is similar to that shown previously in Sec. III and is a convenient check since the image method can be used for comparison. Here, the source is at $30 \mathrm{~m}$ depth and the receiver is at $250 \mathrm{~m}$ range from the source at $40 \mathrm{~m}$ depth. The sea surface is moving at $0.25 \mathrm{~m} / \mathrm{s}$. The same Doppler-sensitive BPSK signal (centered at $9500 \mathrm{~Hz}$ ) as used previously (in Secs. II and III) was the source transmission and the received time series was matched filtered with Doppler adjustments as before. The results for the Kirchhoff time-domain model and the image method are shown in Fig. 17. Note, that this approach does not currently treat receiver motion so the receiver is at a fixed location. 

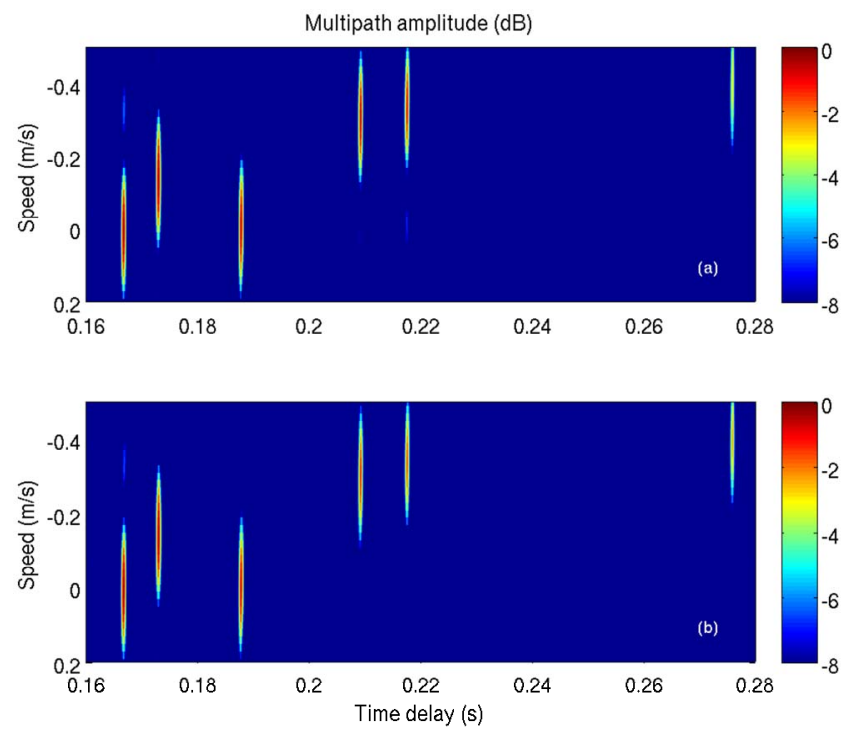

FIG. 17. (Color online) In panel (a), the impulse response (or scattering functions) is computed using the method of images and in (b), it is computed using the time-dependent Kirchhoff approximation method described. The first arrival in time is from the direct path and the third arrival from the bottom bounce both show no Doppler shift. The other paths that follow are from the surface and surface/bottom bounces and are Doppler shifted.

\section{B. Modeling a rough, time-varying surface}

In this example, the sea surface is generated using the Gaussian filtered white noise method and the entire surface is moving at $0.25 \mathrm{~m} / \mathrm{s}$. The standard deviation of the surface is $1.6 \mathrm{~m}$ and the correlation length is $8 \mathrm{~m}$. The surface shape is shown in panel (a) of Fig. 18 and the received time series after applying the Doppler adjusted matched filter to approximate the scattering function in panel (b).

\section{Pulse propagation with a rough surface}

A pulse was transmitted from a source at $40 \mathrm{~m}$ depth using the band from 50 to $600 \mathrm{~Hz}$. The time evolution of this pulse is shown in Fig. 19. This example helps illustrate that
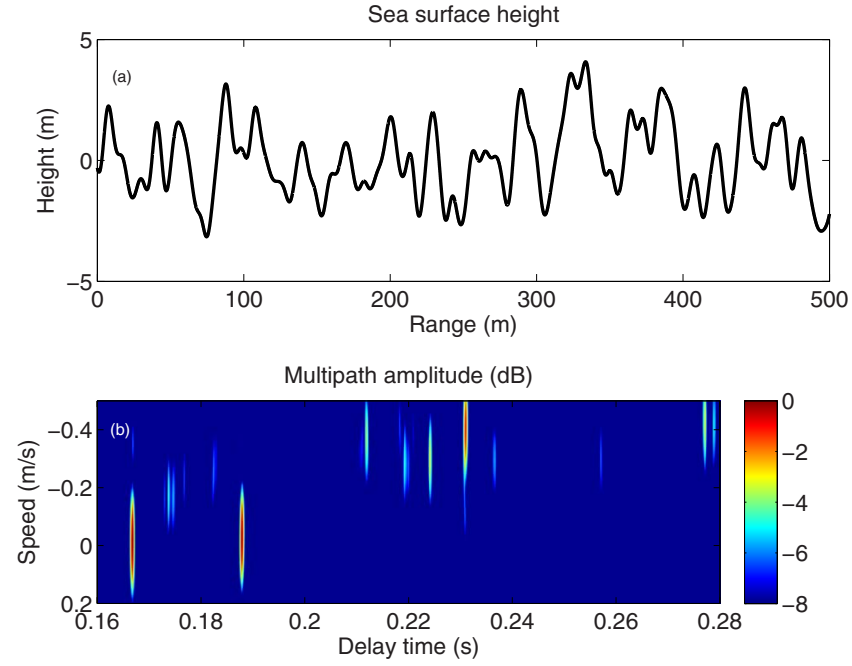

FIG. 18. (Color online) In panel (a), the rough surface is shown for the simulation. This surface moves with a uniform speed of $0.25 \mathrm{~m} / \mathrm{s}$. Shown in (b) is the received time series after applying the Doppler adjusted matched filter to approximate the scattering function.

the model produces the correct pulse shape as well as the reflections from the rough boundary.

\section{CONCLUSION}

Correct scattering and Doppler modeling is important for a variety of underwater acoustic systems such as acoustic modems for communications. The type of communication modulation schemes and data rates depend on the channel spread factor, which is determined by the multipath duration and the Doppler spread. Estimating these quantities is useful for both system design and performance prediction. A binary phase-shift keying communication signal was shown from the Makai experiment, which is typical for that type of ocean environment. The first few arrivals are generally the strongest with the surface interacting paths having Doppler shifts that depend on the surface motion.
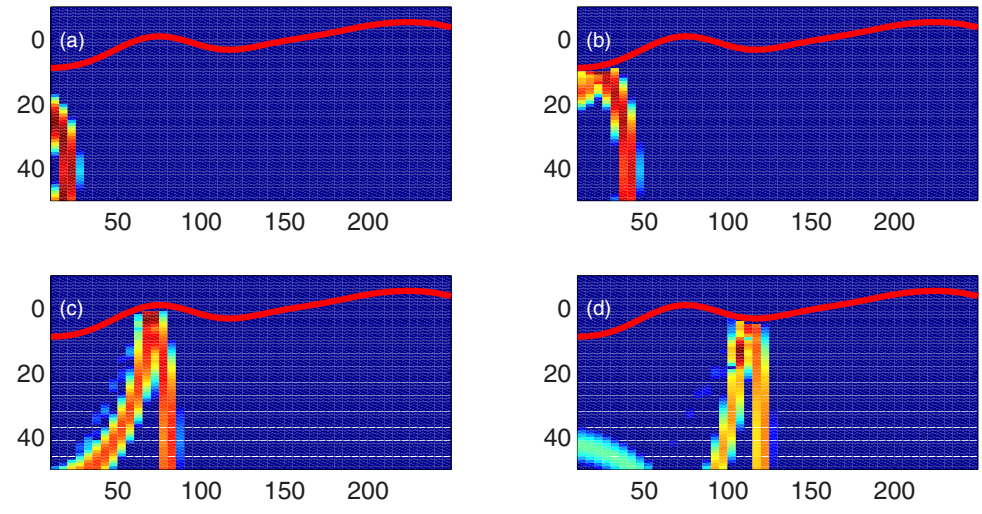

FIG. 19. (Color online) Time evolution of pulse with a rough surface. Times correspond to the following: (a) $0.013 \mathrm{~s}$, (b) $0.026 \mathrm{~s}$, (c) $0.053 \mathrm{~s}$, (d) $0.079 \mathrm{~s}$, (e) $0.11 \mathrm{~s}$, and (f) $0.13 \mathrm{~s}$
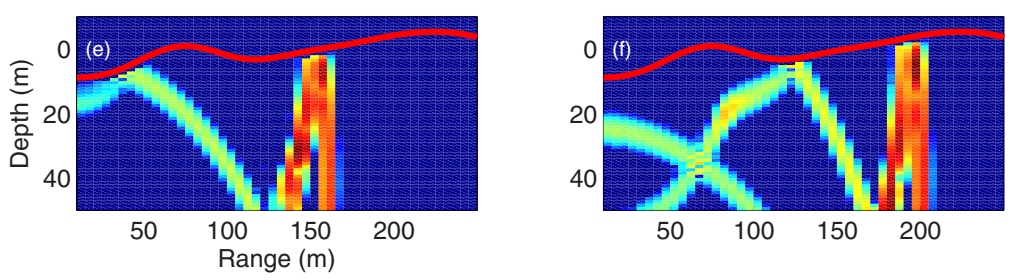
Two simulation methods have been developed for acoustic propagation in the ocean with a time-evolving, rough sea surface. The first method is relatively easy to implement and conveniently allows for receiver motion as well as a changing sea surface. A second method uses the Kirchhoff approximation and was shown to compare well against the exact solutions. There are a number of approximations within this approach including the limitation to six arrivals. However, the approach produces results that are useful for determining how each path is modified by interaction with the rough, time-evolving sea surface.

\section{ACKNOWLEDGMENTS}

The authors are grateful for the support provided for this work by the Office of Naval Research. The authors would like to thank Keyko McDonald (SPAWAR) for the development and deployment of the Telesonar Testbeds used for the experimental data. The authors would also like to thank Sergio Jesus, Antonio da Silva, and Friedrich Zabel at the Signal Processing Laboratory at the University of Algarve, Portugal for their support and cooperation with the received AOB data used for this analysis. The authors would also like to acknowledge their colleagues: Katherine Kim for her help during the Makai experiment, Paul Hursky for his advice on communication topics and his assistance during the Makai experiment, and Ahmad Abawi for many valuable discussions.
${ }^{1}$ M. Siderius and M. B. Porter, "Modeling techniques for marine mammal risk assessment," IEEE J. Ocean. Eng. 31, 49-60 (2006).

${ }^{2}$ M. B. Porter, "The Makai experiment: High-frequency acoustics," in Proceedings of the Eighth European Conference on Underwater Acoustics (Tipografia Uniao, Algarve, Portugal, 2006), pp. 9-18.

${ }^{3}$ V. K. McDonald, P. Hursky, and the KauaiEx Group, "Telesonar testbed instrument provides a flexible platform for acoustic propagation and communication research in the $8-50 \mathrm{kHz}$ band," in Proceedings of the HighFrequency Ocean Acoustics Conference (AIP, Melville, NY, 2004), pp. 336-349.

${ }^{4}$ A. Silva, F. Zabel, and C. Martins, "The acoustic oceanographic buoy telemetry system-A modular equipment that meets acoustic rapid environmental assessment requirements," Sea Technol. 47(9), pp. 15-20 (2006).

${ }^{5}$ J. G. Proakis, Digital Communications, 3rd ed. (McGraw-Hill, New York, NY, 1995).

${ }^{6}$ W. S. Burdic, Underwater Acoustic System Analysis (Prentice-Hall, Englewood Cliffs, NJ, 1991).

${ }^{7}$ R. S. Keiffer, J. C. Novarini, and R. W. Scharstein, "A time-varient impulse response method for acoustic scattering from moving twodimensional surfaces," J. Acoust. Soc. Am. 118, 1283-1299 (2005).

${ }^{8}$ P. C. Etter, Underwater Acoustic Modeling, 2nd ed. (EFN Spon, London, UK, 1996).

${ }^{9}$ F. B. Jensen, W. A. Kuperman, M. B. Porter, and H. Schmidt, Computational Ocean Acoustics (AIP, New York, 1994).

${ }^{10}$ M. B. Porter, Ocean Acoustics Library, Bellhop (http:// oalib.hlsresearch.com/, viewed April 10, 2008).

${ }^{11}$ M. B. Porter and H. P. Bucker, "Gaussian beam tracing for computing ocean acoustic fields," J. Acoust. Soc. Am. 82, 1349-1359 (1987).

${ }^{12}$ J. A. Ogilvy, Theory of Wave Scattering from Random Rough Surfaces (IOP, London, UK, 1991).

${ }^{13}$ E. I. Thorsos, "The validity of the Kirchhoff approximation for rough surface scattering using a Gaussian roughness spectrum," J. Acoust. Soc. Am. 83, 78-92 (1988).

${ }^{14}$ E. Hecht and A. Zajac, Optics (Addison-Wesley, Reading, MA, 1979). 